\title{
A GINZBURG-LANDAU TYPE MODEL OF SUPERCONDUCTING/NORMAL JUNCTIONS INCLUDING JOSEPHSON JUNCTIONS
}

\author{
S. JONATHAN CHAPMAN $\dagger$, QIANG DU $\ddagger$ AND MAX D. GUNZBURGER§
}

\begin{abstract}
A model for superconductors co-existing with normal materials is presented. The model, which applies to such situations as superconductors containing normal impurities and superconductor/normal material junctions, is based on a generalization of the Ginzburg-Landau model for superconductivity. After presenting the model, it is shown that it reduces to well-known models due to de Gennes for certain superconducting/normal interfaces, and in particular, for Josephson junctions. A provident feature of the modified model is that it can, by itself, account for all of these as well as other physical situations. The results of some preliminary computational experiments using the model are then provided; these include flux pinning by normal impurities and a superconductor/normal/superconductor junction. A side benefit of the modified model is that, through its use, these computational simulations are more easily obtained.
\end{abstract}

\section{Introduction}

Samples consisting of both non-superconducting (normal) and superconducting materials are of interest in both the study of superconducting phenomena and the design of superconducting devices. For example, it is widely accepted that normal impurities in an otherwise superconducting material provide pinning sites for vortices, and thus prevent their motion and the resultant induced resistivity. Another example is, of course, the Josephson junction in which a thin layer of normal material is sandwiched between layers of superconducting material.

When a normal material is placed adjacent to a superconducting material, two phenomena are observed, which are collectively known as the proximity effect. Firstly, the presence of the normal material hampers the pairing of electrons in the superconducting material, so that the number density of superconducting electron pairs is reduced near the boundary to a value below that of the bulk. Secondly, the presence of the superconducting material promotes the formation of electron pairs in the normal material, so that there is a nonzero density of superconducting electron pairs in the normal material near the boundary; see Figure 1.1. The strength of each effect is determined by the nature of the normal material.

In the Ginzburg-Landau theory of superconductivity, these phenomena are usually modelled through the boundary conditions (see, e.g., [4]) on the superconducting order parameter at the normal/superconducting interface. There are several drawbacks with this approach. Firstly, there is only one parameter in the boundary condition to describe the normal material, so that the two phenomena alluded to above cannot be separated: a normal material which does not break the superconducting electron pairs in the superconducting material is forced to also have a large number density of superconducting electron pairs induced in itself. (An extreme situation in

†Mathematical Institute, University of Oxford, 24-29 St. Giles', Oxford, OX1 3LB, UK.

$\ddagger$ Department of Mathematics, Michigan State University, East Lansing, MI 48824, USA.

$\S$ Department of Mathematics, Virginia Tech, Blacksburg, VA 24061, USA. Supported in part by the Air Force Office of Scientific Research under grant number AFOSR-93-1-0061. 
which this is clearly not the case is that in which the non-superconducting material is in fact a vacuum, which has no effect on the superconducting electron pairs in the superconducting material, but which also can have no induced superconducting electron pairs itself.) Related to this is the second drawback, which is that the boundary conditions only allow for the calculation of the supercurrent in the superconducting material - the normal material is ignored. However, a complete solution involves a coupling with the magnetic field through Maxwell's equations in the normal material. In this case any supercurrent in the normal material will affect the magnetic field and so alter the solution. Thirdly, the boundary condition used implies that no current may pass through the boundary. Since there is a nonzero density of superconducting electron pairs in the normal material, there seems to be no reason to impose such a condition, particularly in cases with a lack of symmetry.

FIG. 1.1. The proximity effect; in the superconducting region, the density is lower than the bulk value; in the normal region, the density is non-zero.

In this paper we study a different approach, still within the framework of the Ginzburg-Landau theory, to modelling superconducting/normal junctions. We will find that in some cases the model reduces to the usual boundary condition, although allowing the supercurrent in the normal material to be calculated. However, in other cases it will not result in this boundary condition, and may lead to a current passing through the interface. The model can be used to describe various types of interactions involving normal/superconducting materials, including Josephson junctions and the effects of normal impurities within a superconducting material.

The model also results in a substantial simplification of computer simulations involving normal/superconducting samples. Instead of solving a Ginzburg-Landau model in the superconductor coupled with a Maxwell model in normal regions, one now merely employs a single model throughout the sample.

The layout of the paper is as follows. In $\S 2$, we introduce the Ginzburg-Landau theory of superconductivity. In $\S 3$, we modify this theory to obtain a better description of the proximity effect, and we show that the modified approach does in fact reduce to the old approach both in one-dimension and when the region external to the superconductor is a vacuum. In $\S 4$, we examine a planar superconducting/normal/superconducting Josephson junction using the modified model, and find that it results in the usual expression for the tunnelling current. Finally, in $\S 5$, we provide the results of some computational experiments utilizing the modified model.

We note that one feature of the modified model has been previously proposed in [3]. We discuss this further in $\S 3$ where the modified model is presented. 


\section{The Ginzburg-Landau model}

In [2]. Ginzburg and Landau postulated that the Gibbs free energy of a superconducting material should be given by

$$
\mathcal{G}_{s}=\int_{\Omega_{s}}\left\{f_{s}+a|\tilde{\psi}|^{2}+\frac{b}{2}|\tilde{\psi}|^{4}+\frac{1}{2 \tilde{m}_{s}}\left|\left(i \hbar \nabla+\frac{e_{s}}{c} \tilde{\mathbf{A}}\right) \tilde{\psi}\right|^{2}+\frac{\tilde{\mu}_{s}}{8 \pi} \tilde{\mathbf{H}} \cdot\left(\tilde{\mathbf{H}}-2 \tilde{\mathbf{H}}_{0}\right)\right\} d \Omega,
$$

where $f_{s}$ is the free energy density of the non-superconducting state in the absence of a magnetic field, $\tilde{\psi}$ is the (complex-valued) superconducting order parameter, $\tilde{\mathbf{A}}$ is the magnetic vector potential, $\tilde{\mathbf{H}}=\left(1 / \tilde{\mu}_{s}\right) \operatorname{curl} \tilde{\mathbf{A}}$ is the magnetic field, $\tilde{\mathbf{H}}_{0}$ is the applied magnetic field, $a$ and $b$ are constants whose values depend on the temperature and such that $b>0, e_{s}$ is mass of the superconducting charge carriers which is twice the electronic charge $e, c$ is the speed of light, $\tilde{\mu}_{s}$ is the permeability, $2 \pi \hbar$ is Planck's constant, and $\Omega_{s}$ is the (bounded) region occupied by the superconducting material. The mass $\tilde{m}_{s}$ is in fact arbitrary within the framework of the phenomenological theory (modifying $\tilde{m}_{s}$ simply scales $\tilde{\psi}, a$, and $b$ ) but is usually taken to be the twice the electron mass. We will consider only temperatures below the critical temperature of the superconductor, in which case $a<0$.

The basic postulate of the Ginzburg-Landau theory is that the total Gibbs free energy is minimized. Before we perform the minimization we must add to the Gibbs free energy of the superconducting material the Gibbs free energy of the region external to the superconductor, which we denote by $\Omega_{n}$, due to the magnetic field there:

$$
\mathcal{G}_{n}=\int_{\Omega_{n}}\left\{f_{n}+\frac{\tilde{\mu}_{n}}{8 \pi} \tilde{\mathbf{H}} \cdot\left(\tilde{\mathbf{H}}-\tilde{\mathbf{H}}_{0}\right)\right\} d \Omega .
$$

Note that the permeability $\tilde{\mu}$ may take different values in the superconducting and normal regions. Also, $\Omega_{n}$ may itself consist of of subregions, each occupied by different materials, e.g., a metal and/or a vacuum.

Minimizing the total Gibbs free energy with respect to $\tilde{\psi}^{*}$, the conjugate of $\tilde{\psi}$, and $\tilde{\mathbf{A}}$, we obtain the celebrated Ginzburg-Landau equations

$$
\begin{gathered}
\frac{1}{2 \tilde{m}_{s}}\left(i \hbar \nabla+\frac{e_{s}}{c} \tilde{\mathbf{A}}\right)^{2} \tilde{\psi}+a \tilde{\psi}+b|\tilde{\psi}|^{2} \tilde{\psi}=0 \quad \text { in } \Omega_{s} \\
\frac{1}{4 \pi \tilde{\mu}_{s}}(\mathbf{c u r l})^{2} \tilde{\mathbf{A}}=-\frac{i e_{s} \hbar}{2 c \tilde{m}_{s}}\left(\tilde{\psi}^{*} \nabla \tilde{\psi}-\tilde{\psi} \nabla \tilde{\psi} \tilde{\psi}^{*}\right)-\frac{e_{s}^{2}}{c^{2} \tilde{m}_{s}}|\tilde{\psi}|^{2} \tilde{\mathbf{A}} \quad \text { in } \Omega_{s} \\
(\operatorname{curl})^{2} \tilde{\mathbf{A}}=\mathbf{0} \quad \text { in } \Omega_{n}, \\
\mathbf{n} \cdot\left(i \hbar \nabla+\frac{e_{s}}{c} \tilde{\mathbf{A}}\right) \tilde{\psi}=0 \quad \text { on } \partial \Omega_{s} \\
{\left[\frac{1}{\tilde{\mu}} \operatorname{curl} \tilde{\mathbf{A}} \times \mathbf{n}\right]=\mathbf{0}}
\end{gathered}
$$$$
[\tilde{\mathbf{A}}]=\mathbf{0},
$$

and, if $\Omega_{n}$ contains an exterior domain,

$$
\operatorname{curl} \tilde{\mathbf{A}} \rightarrow \tilde{\mu}_{n} \tilde{\mathbf{H}}_{0} \quad \text { as }|\tilde{\mathbf{x}}| \rightarrow \infty,
$$


where $\partial \Omega_{s}$ denotes the boundary of the region $\Omega_{s}$ occupied by the superconductor, $[\cdot]$ denotes the jump in the enclosed quantity across $\partial \Omega_{s}$, and $|\tilde{\mathbf{x}}|$ denotes the distance from the origin. We note that these equations can also be derived as a suitable limit of the microscopic (BCS) theory of superconductivity, and that this will evaluate the parameters $a, b, m_{s}$, etc. in terms of parameters appearing in the microscopic equations. We employ the usual nondimensionalizations:

$$
\tilde{\psi}=\sqrt{\frac{|a|}{b}} \psi, \quad \tilde{\mathbf{H}}=\sqrt{\frac{8 \pi a^{2}}{b \tilde{\mu}_{s}}} \mathbf{H}, \quad \tilde{\mathbf{A}}=\sqrt{\frac{2|a| c^{2} \tilde{m}_{s}}{e_{s}^{2}}} \mathbf{A}, \quad \text { and } \quad \tilde{\mathbf{x}}=\sqrt{\frac{\tilde{m}_{s} b c^{2}}{4 \pi|a| e_{s}^{2} \tilde{\mu}_{s}}} \mathbf{x}
$$

giving

$$
\begin{gathered}
\left(\frac{i}{\kappa} \nabla+\mathbf{A}\right)^{2} \psi-\psi+|\psi|^{2} \psi=0 \quad \text { in } \Omega_{s} \\
(\text { curl })^{2} \mathbf{A}=-\frac{i}{2 \kappa}\left(\psi^{*} \nabla \psi-\psi \nabla \psi^{*}\right)-|\psi|^{2} \mathbf{A} \quad \text { in } \Omega_{s} \\
(\operatorname{curl})^{2} \mathbf{A}=\mathbf{0} \quad \text { in } \Omega_{n} \\
\mathbf{n} \cdot\left(\frac{i}{\kappa} \nabla+\mathbf{A}\right) \psi=0 \quad \text { on } \partial \Omega_{s} \\
{\left[\frac{1}{\mu} \operatorname{curl} \mathbf{A} \times \mathbf{n}\right]=\mathbf{0}}
\end{gathered}
$$

and, if $\Omega_{n}$ contains an exerior domain,

$$
\operatorname{curl} \mathbf{A} \mu_{n} \rightarrow \mathbf{H}_{0} \quad \text { as } \mathbf{x} \rightarrow \infty
$$

where

$$
\kappa=\sqrt{\frac{\tilde{m}_{s}^{2} c^{2} b}{2 \pi \tilde{\mu}_{s} e_{s}^{2} \hbar^{2}}}
$$

denotes the Ginzburg-Landau parameter and $\mu=\tilde{\mu} / \tilde{\mu}_{s}$ so that $\mu_{s}=1$ and $\mu_{n}=$ $\tilde{\mu}_{n} / \tilde{\mu}_{s}$.

The boundary condition (2.4) is the natural boundary condition associated with the minimization process, and corresponds to the superconductor being adjacent to a vacuum. For an interface between a superconducting material and another material, de Gennes (see [1]) has proposed a modification of this boundary condition to

$$
\mathbf{n} \cdot\left(\frac{i}{\kappa} \nabla+\mathbf{A}\right) \psi=-i \gamma \psi \quad \text { on } \partial \Omega_{s},
$$


where $\gamma$ is a real, positive parameter. This relation is derived from the BCS theory for a planar normal/superconducting interface in the absence of a magnetic field (so that $A=0$ and $\psi$ is real) by assuming (i) that no current passes through the boundary, and (ii) that there exists a local boundary condition of the form $f\left(\psi, \psi_{n}, \psi_{n n}, \cdots\right)=0$. Since higher derivatives can be expressed in terms of $\psi$ and $\psi_{n}$ using the GinzburgLandau equations, such a condition can be written in the form

$$
\psi_{n}=-\gamma \psi-\delta \psi^{3}+\cdots .
$$

The BCS theory implies that only odd powers of $\psi$ appear in the expansion, and can also be used to evaluate the coefficients $\gamma, \delta$, etc. The $\psi^{3}$ term is shown to be negligible in the regime of validity of the Ginzburg-Landau equations, to give

$$
\psi_{n}=-\gamma \psi \text {. }
$$

In the presence of a magnetic field, and for curved boundaries, this is replaced by (2.8), in which the derivative has simply been made gauge invariant.

Equation (2.8) describes the proximity effect in the sense shown in Figure 2.1. If the order parameter is extended into the normal material linearly from its value and slope on the boundary, then it penetrates a distance $1 /(\kappa \gamma)$ before reaching zero. This is taken to be the extent to which the superconducting electrons penetrate the normal material. (Notice that if the same interpretation is applied to the boundary condition (2.4) when the superconductor is adjacent to a vacuum, we would find a large density of superconducting electrons throughout the vacuum region, which is obviously absurd.) Note that the number density of superconducting electrons is reduced near the interface. For insulating materials, $\gamma$ is supposed to be small, so that $|\psi|$ is close to its value in the bulk material. For magnetic materials, there is a large disruption of the superconducting electron pairs in the superconducting material, and $\gamma$ is supposed to be large so that $|\psi|$ is small on the boundary.

FIG. 2.1. The proximity effect: straight line extension of the order parameter into the normal region.

We note that (2.2) implies that the (nondimensionalized) supercurrent $\mathbf{J}=\operatorname{curl} \mathbf{H}$ is given by

$$
\mathbf{J}=-\frac{i}{2 \kappa}\left(\psi^{*} \nabla \psi-\psi \nabla \psi^{*}\right)-|\psi|^{2} \mathbf{A}=-\frac{1}{2}\left\{\psi^{*}\left(\frac{i}{\kappa} \nabla+\mathbf{A}\right) \psi+\psi\left(-\frac{i}{\kappa} \nabla+\mathbf{A}\right) \psi^{*}\right\}
$$

so that (2.8) implies that $\mathbf{J} \cdot \mathbf{n}=0$ on $\partial \Omega_{s}$, i.e., no current flows through the boundary of the superconductor. 


\section{The modified Ginzburg-Landau model}

In this section we reformulate the Gibbs free energy for the normal material. Instead of simply minimizing the energy due to the magnetic field, we now allow for the possibility of a nonzero density of superconducting electrons. We let the normal material be capable of becoming superconducting, and be described by a free energy functional similar to that of the superconducting material. However, the parameters in the two functionals may be different. In particular, we will assume that the normal material is above its critical temperature, and so the corresponding coefficient $a$ will be positive. (We note that the procedure could equally well be applied to a boundary between different superconductors, in which the coefficient $a$ would still be negative.) Other coefficients which will change in the normal material are $b$ (which will henceforth, for simplicity, be taken to be zero since with $a$ positive the free energy functional is convex and the effect of nonzero $b$ will presumably be a minor perturbation), $\mu$, and $m$, which, as we have said, is arbitrary in the Ginzburg-Landau model.

The use of positive values of the parmeter $a$ to model normal metals within the framework of the Ginzburg-Landau free energy functional has been previously proposed in [3]. Here, we show that if in addition one chooses appropriate values for the mass parameter $m$ in non-superconducting regions, one can then model other types of normal regions within the Ginzburg-Landau framework.

The total Gibbs free energy is now given by

$$
\begin{gathered}
\int_{\Omega_{s}}\left\{f_{s}+a_{s}|\tilde{\psi}|^{2}+\frac{b_{s}}{2}|\tilde{\psi}|^{4}+\frac{1}{2 \tilde{m}_{s}}\left|\left(i \hbar \nabla+\frac{e_{s}}{c} \tilde{\mathbf{A}}\right) \tilde{\psi}\right|^{2}+\frac{\tilde{\mu}_{s}}{8 \pi}|\tilde{\mathbf{H}}|^{2}-\frac{\tilde{\mu}_{s}}{4 \pi} \tilde{\mathbf{H}} \cdot \tilde{\mathbf{H}}_{0}\right\} d \Omega \\
+\int_{\Omega_{n}}\left\{f_{n}+a_{n}|\tilde{\psi}|^{2}+\frac{1}{2 \tilde{m}_{n}}\left|\left(i \hbar \nabla+\frac{e_{n}}{c} \tilde{\mathbf{A}}\right) \tilde{\psi}\right|^{2}+\frac{\tilde{\mu}_{n}}{8 \pi}|\tilde{\mathbf{H}}|^{2}-\frac{\tilde{\mu}_{n}}{4 \pi} \tilde{\mathbf{H}} \cdot \tilde{\mathbf{H}}_{0}\right\} d \Omega
\end{gathered}
$$

which, on minimizing, leads to

$$
\begin{gathered}
\frac{1}{2 \tilde{m}_{s}}\left(i \hbar \nabla+\frac{e_{s}}{c} \tilde{\mathbf{A}}\right)^{2} \tilde{\psi}+a_{s} \tilde{\psi}+b_{s}|\tilde{\psi}|^{2} \tilde{\psi}=0 \quad \text { in } \Omega_{s}, \\
\frac{1}{4 \pi \tilde{\mu}_{s}}(\mathbf{c u r l})^{2} \tilde{\mathbf{A}}=-\frac{i e_{s} \hbar}{2 c \tilde{m}_{s}}\left(\tilde{\psi} * \nabla \tilde{\psi}-\tilde{\psi} \nabla \tilde{\psi} \tilde{\psi}^{*}\right)-\frac{e_{s}^{2}}{c^{2} \tilde{m}_{s}}|\tilde{\psi}|^{2} \tilde{\mathbf{A}} \quad \text { in } \Omega_{s}, \\
\frac{1}{2 \tilde{m}_{n}}\left(i \hbar \nabla+\frac{e_{n}}{c} \tilde{\mathbf{A}}\right)^{2} \tilde{\psi}+a_{n} \tilde{\psi}=0 \quad \text { in } \Omega_{n}, \\
\frac{1}{4 \pi \tilde{\mu}_{n}}(\mathbf{c u r l})^{2} \tilde{\mathbf{A}}=-\frac{i e_{n} \hbar}{2 c \tilde{m}_{n}}\left(\tilde{\psi} * \nabla \tilde{\psi}-\tilde{\psi} \nabla \tilde{\psi}^{*}\right)-\frac{e_{n}^{2}}{c^{2} \tilde{m}_{n}}|\tilde{\psi}|^{2} \tilde{\mathbf{A}} \quad \text { in } \Omega_{n}, \\
{[\tilde{\mathbf{A}}]=\mathbf{0}, \quad[\tilde{\psi}]=0,} \\
{\left[\mathbf{n} \cdot \frac{1}{\tilde{m}}\left(i \hbar \nabla+\frac{e}{c} \tilde{\mathbf{A}}\right) \tilde{\psi}\right]=0,}
\end{gathered}
$$

and

$$
\left[\frac{1}{\tilde{\mu}} \operatorname{curl} \tilde{\mathbf{A}} \times \mathbf{n}\right]=\mathbf{0} .
$$

As before, $e_{s}$ and $e_{n}$ are equal to the charge of the superconducting charge carriers, and are both equal to twice the electron charge. Of the masses $\tilde{m}_{n}$ and $\tilde{m}_{s}$ we are free to choose one arbitrarily. We again choose $\tilde{m}_{s}$ to be twice the electron mass. This leaves 
$\tilde{m}_{n}$ as a parameter depending upon the normal material. The dependence of $\tilde{m}_{n}$ and $\tilde{a}_{n}$ on the type of normal material can be determined either phenomenologically, or, as we have said, from the microscopic theory. Since the Ginzburg-Landau equations are widely used as phenomenological equations outside their strict range of validity as a limiting form of the microscopic theory we adopt the former viewpoint. However, we note that in particular the microscopic theory implies that $\tilde{m}_{n} \propto 1 / \sigma_{n}$, where $\sigma_{n}$ is the (normal) conductivity of the normal region. Using the values of the parameters in the superconducting material to effect the nondimensionalization as before leads to:

$$
\left[\mathbf{n} \cdot \frac{1}{m}\left(\frac{i}{\kappa} \nabla+\mathbf{A}\right) \psi\right]=0
$$

and

$$
\left[\frac{1}{\mu} \operatorname{curl} \mathbf{A} \times \mathbf{n}\right]=0
$$

where $m=\tilde{m} / \tilde{m}_{s}$ and $\mu=\tilde{\mu} / \tilde{\mu}_{s}$ (so that $m_{s}=\mu_{s}=1$ ), and $\alpha=\tilde{m}_{n} a_{n} /\left(\tilde{m}_{s}\left|a_{s}\right|\right)$.

FIG. 3.1. The proximity effect with continuous or discontinuous normal derivative at the interface. The dashed curve for a continuous derivative must penetrate deep into the normal region. The solid curve for a discontinuous derivative need not peneterate as much. 
Note that the single parameter $\gamma$ of the de Gennes boundary condition (2.8) has been replaced by two parameters, $\alpha$ and $m_{n}$, which will depend on the normal material. Also, note that the normal derivative of $\psi$ is not required to be continuous, so that a low pairbreaking strength does not necessarily imply a large penetration; see Figure 3.1. Note that the (nondimensionalized) current in the normal region is now given by

$$
\mathbf{J}=-\frac{1}{m_{n}}\left(\frac{i}{2 \kappa}\left(\psi^{*} \nabla \psi-\psi \nabla \psi^{*}\right)+|\psi|^{2} \mathbf{A}\right) .
$$

We now consider the behavior of the equations for various values of the parameters. In particular, we wish to verify that the model is consistent with the de Gennes theory in situations in which that theory has been justified microscopically.

\subsection{One-dimension}

In one-dimension, in the absence of a magnetic field, we expect the theory to be able to recover the de Gennes boundary condition (2.8). In the one-dimensional setting, the equations in the normal region $x>0$ are given by

$$
\frac{1}{\kappa^{2}} \psi^{\prime \prime}=\alpha \psi+A^{2} \psi \quad \text { and } \quad A^{\prime \prime}=\frac{\mu_{n}}{m_{n}} \psi^{2} A,
$$

where $^{\prime} \equiv d / d x$. At $x=0$, we have the interface conditions

$$
[A]=0, \quad[\psi]=0, \quad\left[\frac{1}{\mu} A^{\prime}\right]=0, \quad \text { and } \quad\left[\frac{1}{m} \psi^{\prime}\right]=0 .
$$

Let us consider the case $A=0$. Then, in the normal region $x>0$, we have

$$
\psi^{\prime \prime}=\kappa^{2} \alpha \psi
$$

so that

$$
\psi=\psi\left(0^{+}\right) e^{-\kappa \sqrt{\alpha} x} .
$$

Therefore,

$$
\psi^{\prime}\left(0^{+}\right)=-\kappa \sqrt{\alpha} \psi\left(0^{+}\right) .
$$

Hence, using the interface conditions (3.8), we have, on the superconducting side of the boundary,

$$
\psi^{\prime}\left(0^{-}\right)=-\frac{\kappa \sqrt{\alpha}}{m_{n}} \psi\left(0^{-}\right) .
$$

Thus, we retrieve de Gennes boundary condition (2.8) with $\gamma=\sqrt{\alpha} / m_{n}$.

When $A \neq 0$ we can still in principle solve the equations in the region $x>0$ and relate $\psi^{\prime}\left(0^{+}\right)$to $\psi\left(0^{+}\right)$. However, in this case the relationship will not be linear, and will depend on the applied magnetic field. The reason for the increased complexity is the interaction between $\psi$ and $A$ via the supercurrent in the normal region, which was not taken into account in deriving (2.8).

\subsection{Vacuum}

We also expect to be able to retrieve the classical Ginzburg-Landau boundary conditions for an interface with a vacuum. For a vacuum, we should have $\psi=0$, and 
this can be achieved by letting $\alpha \rightarrow \infty$ in equation (3.3), which gives $\psi \rightarrow 0$ except in thin boundary layers. This now implies that the gradient of $\psi$ is becoming large on the normal side of the boundary, so that in order to retrieve the boundary condition (2.5) on the superconducting side of the boundary we must also let $m_{n} \rightarrow \infty$. (Note that this is in agreement with $\sigma_{n}=0$.) Equation (3.3) implies that the gradient of $\psi$ grows as $\sqrt{\alpha}$, hence to retrieve (2.5) $m_{n}$ must grow more quickly than $\sqrt{\alpha}$.

Thus, we see that the limit $\alpha \rightarrow \infty, m_{n} \rightarrow \infty$ in such a way that $\sqrt{\alpha} / m_{n} \rightarrow 0$ corresponds to the non-superconducting region being a vacuum. Equations (3.4) and (3.6) then reduce to equations (2.3) and (2.5) respectively, and $\psi$ is equal to zero in the vacuum region, so that $\psi$ is discontinuous in the limit.

In general, the limit $m_{n} \rightarrow \infty, \sqrt{\alpha} / m_{n} \rightarrow 0$ will lead to (2.3) and (2.5), and the current in the non-superconducting region will be zero whatever the value of $\psi$; in this sense, $\psi$ in the normal region is irrelevant when $m_{n}=\infty$.

\section{Josephson junctions}

We now consider a planar S-N-S Josephson junction. In [1], De Gennes used the Ginzburg-Landau theory to model such a junction in the following way. The values of $\psi$ and $\mathbf{n} \cdot\left(\frac{i}{\kappa} \nabla+\mathbf{A}\right) \psi$ as the normal region is approached from the right-hand superconducting side [denoted by $(\cdot)^{+}$] are taken to be related to their values as the junction is approached from the left-hand superconducting side [denoted by $\left.(\cdot)^{-}\right]$. This relationship is linearized to give

$$
\left(\begin{array}{c}
\psi \\
\mathbf{n} \cdot\left(\frac{1}{\kappa} \nabla-i \mathbf{A}\right) \psi
\end{array}\right)^{+}=\left(\begin{array}{ll}
M_{11} & M_{12} \\
M_{21} & M_{22}
\end{array}\right)\left(\begin{array}{c}
\psi \\
\mathbf{n} \cdot\left(\frac{1}{\kappa} \nabla-i \mathbf{A}\right) \psi
\end{array}\right)^{-}
$$

where the $M_{i j}$ are real and are determined by the particular junction and depend on its thickness, the type of material, etc.

The supercurrent across the junction is then given by

$$
J=\frac{1}{M_{12}}\left|\psi^{+} \psi^{-}\right| \sin \left(\chi^{+}-\chi^{-}\right)
$$

where $\psi=|\psi| e^{i \chi}$. (Note that the previously used boundary condition (2.8) at a normal/superconducting interface now does not hold, since that condition would imply no current through the junction.) Then, de Gennes postulates that for an insulating material $M_{11}=M_{22}=1$, and $M_{12}$ and $M_{21}$ are small. The junction is symmetric if and only if $M_{11}=M_{22}$. In this case (see [1]),

$$
M_{11} M_{22}-M_{12} M_{21}=1 .
$$

Here we model such a junction by the modified Ginzburg-Landau approach introduced in $\S 3$. We consider a junction of small but nonzero thickness $2 d$; see the sketch in Figure 4.1. We will find that for $d \ll 1$ we do in fact retrieve the condition (4.1), and that we can evaluate the coefficients $M_{i j}$ in terms of the junction parameters. 
FIG. 4.1. A superconducting-normal-superconducting junction of thickness $2 d$.

We have the following equations: in the left superconducting region $x<-d$ we have

$$
\left(\frac{i}{\kappa} \nabla+\mathbf{A}\right)^{2} \psi-\psi+|\psi|^{2} \psi=0
$$

and

$$
(\mathbf{c u r l})^{2} \mathbf{A}=-\frac{i}{2 \kappa}\left(\psi^{*} \nabla \psi-\psi \nabla \psi^{*}\right)-|\psi|^{2} \mathbf{A} ;
$$

in the normal region $-d \leq x \leq d$ we have

$$
\left(\frac{i}{\kappa} \nabla+\mathbf{A}\right)^{2} \psi+\alpha \psi=0
$$

and

$$
(\operatorname{curl})^{2} \mathbf{A}=-\frac{\mu_{n}}{m_{n}}\left\{\frac{i}{2 \kappa}\left(\psi^{*} \nabla \psi-\psi \nabla \psi^{*}\right)+|\psi|^{2} \mathbf{A}\right\} ;
$$

in the right superconducting region we have

$$
\left(\frac{i}{\kappa} \nabla+\mathbf{A}\right)^{2} \psi+a_{s_{2}} \psi+b_{s_{2}}|\psi|^{2} \psi=0
$$

and

$$
(\operatorname{curl})^{2} \mathbf{A}=-\frac{\mu_{s_{2}}}{m_{s_{2}}}\left\{\frac{i}{2 \kappa}\left(\psi^{*} \nabla \psi-\psi \nabla \psi^{*}\right)+|\psi|^{2} \mathbf{A}\right\} ;
$$

we also have the interface conditions at both $x=-d$ and $x=d$

$$
\begin{gathered}
{[\mathbf{A}]=0, \quad[\psi]=0,} \\
{\left[\frac{1}{\mu} \operatorname{curl} \mathbf{A}\right]=0,}
\end{gathered}
$$

and

$$
\left[\frac{1}{m}\left(\frac{i}{\kappa} \nabla+\mathbf{A}\right) \psi\right]=0
$$

Here we have allowed the possibility of having a different superconducting material in $x>d$ to that in $x<-d$, and have used the parameters refering to the material in $x<-d$ for the nondimensionalization, so that $m_{s_{1}}=\mu_{s_{1}}=b_{s_{1}}=-a_{s_{1}}=1$. 
By solving the equations in the normal region, we now analyse this model to determine the supercurrent through the junction, and to retrieve the de Gennes condition (4.1). We rescale lengths with $d$ by setting $\mathbf{x}=d \mathbf{X}$ to obtain

$$
\left(\frac{i}{d \kappa} \nabla \mathbf{X}+\mathbf{A}\right)^{2} \psi+\alpha \psi=0
$$

and

$$
\left(\operatorname{curl}_{\mathbf{X}}\right)^{2} \mathbf{A}=-\frac{\mu_{n}}{m_{n}} d^{2}\left\{\frac{i}{2 d \kappa}\left(\psi^{*} \nabla_{\mathbf{X}} \psi-\psi \nabla_{\mathbf{X}} \psi^{*}\right)+|\psi|^{2} \mathbf{A}\right\}
$$

in $-1<X<1$. Here, the notation $\nabla_{\mathbf{X}}$ and curl $_{\mathbf{X}}$ serves to remind us that derivatives are with respect to the scaled variable $\mathbf{X}$. We have denoted the first component of $\mathbf{X}$ by $X$. We assume that $d$ is small, and that the current

$$
\mathbf{J}=-\frac{1}{m_{n}}\left\{\frac{i}{2 d \kappa}\left(\psi^{*} \nabla_{\mathbf{X}} \psi-\psi \nabla_{\mathbf{X}} \psi^{*}\right)+|\psi|^{2} \mathbf{A}\right\}
$$

is $o\left(d^{-2}\right)$, so that to a first approximation the vector potential $\mathbf{A}$ is constant. We will return to check the consistency of this assumption later. Let

$$
\psi(\mathbf{X})=e^{i \kappa d \mathbf{A} \cdot \mathbf{X}} \phi(\mathbf{X}) .
$$

(This corresponds to a change in the gauge of the magnetic potential.) Then, (4.4) yields

$$
\frac{1}{\kappa^{2} d^{2}} \Delta_{\mathbf{X}} \phi+\alpha \phi=0
$$

We assume that $\phi$, and thus the modulus of $\psi$, depends only on $X$. The solution of (4.6) is then

$$
\phi=C \cosh (\kappa d \sqrt{\alpha} X)+D \sinh (\kappa d \sqrt{\alpha} X) .
$$

We have four boundary conditions, namely

$$
[\psi]=0 \quad \text { and } \quad\left[\mathbf{n} \cdot \frac{1}{m}\left(\frac{i}{\kappa d} \nabla \mathbf{x}+\mathbf{A}\right) \psi\right]=0 \quad \text { at } \quad X= \pm 1 .
$$

These imply that $\phi$ and $(1 / m) \nabla_{\mathbf{X}} \phi \cdot \mathbf{n}$ are continuous at $X= \pm 1$, or, since we have assumed that $\phi$ is a function of $X$ only, that

$$
[\phi]=0 \quad \text { and } \quad[(1 / m) d \phi / d X]=0 \quad \text { at } X= \pm 1 .
$$

This will allow us to determine $C$ and $D$, and to ultimately relate the values of $\psi$ and $\mathbf{n} \cdot\left(\frac{1}{\kappa} \nabla-i \mathbf{A}\right) \psi$ at $x=d$ to those at $x=-d$. From (4.7), we have

$$
\begin{gathered}
\phi(-1)=C \cosh (\kappa d \sqrt{\alpha})-D \sinh (\kappa d \sqrt{\alpha}), \\
\phi(1)=C \cosh (\kappa d \sqrt{\alpha})+D \sinh (\kappa d \sqrt{\alpha}),
\end{gathered}
$$




$$
\frac{d \phi}{d X}(-1)=-C \kappa d \sqrt{\alpha} \sinh (\kappa d \sqrt{\alpha})+D \kappa d \sqrt{\alpha} \cosh (\kappa d \sqrt{\alpha}),
$$

and

$$
\frac{d \phi}{d X}(1)=C \kappa d \sqrt{\alpha} \sinh (\kappa d \sqrt{\alpha})+D \kappa d \sqrt{\alpha} \cosh (\kappa d \sqrt{\alpha}) .
$$

Solving (4.9) and (4.11) for $C$ and $D$ in terms of the values at $X=-1$ gives

$$
C=\phi(-1) \cosh (\kappa d \sqrt{\alpha})+\frac{d \phi}{d X}(-1) \frac{1}{\kappa d \sqrt{\alpha}} \sinh (\kappa d \sqrt{\alpha})
$$

and

$$
D=\phi(-1) \sinh (\kappa d \sqrt{\alpha})+\frac{d \phi}{d X}(-1) \frac{1}{\kappa d \sqrt{\alpha}} \cosh (\kappa d \sqrt{\alpha}) .
$$

Substituting into (4.10) and (4.12) yields

$$
\phi(1)=\cosh (2 \kappa d \sqrt{\alpha}) \phi(-1)+\frac{\sinh (2 \kappa d \sqrt{\alpha})}{\kappa d \sqrt{\alpha}} \frac{d \phi}{d X}(-1)
$$

and

$$
\frac{d \phi}{d X}(1)=\kappa d \sqrt{\alpha} \sinh (2 \kappa d \sqrt{\alpha}) \phi(-1)+\cosh (2 \kappa d \sqrt{\alpha}) \frac{d \phi}{d X}(-1) .
$$

Returning to the original gauge and length scale, we find that since

$$
\left(\frac{1}{\kappa} \frac{d}{d x}-i \mathbf{A}\right) \psi=\left(\frac{1}{\kappa d} \frac{d}{d X}-i \mathbf{A}\right) \psi=\frac{1}{\kappa d} e^{i \kappa d \mathbf{A} \cdot \mathbf{x}}\left(\frac{d \phi}{d X}\right),
$$

then

$$
\begin{aligned}
& \left.\left(\begin{array}{c}
\psi \\
\left(\frac{1}{\kappa} \frac{d}{d x}-i A\right) \psi
\end{array}\right)\right|_{d_{-}} \\
& \quad=\left.\left(\begin{array}{cc}
\cosh (2 \kappa d \sqrt{\alpha}) & \sinh (2 \kappa d \sqrt{\alpha}) / \sqrt{\alpha} \\
\sqrt{\alpha} \sinh (2 \kappa d \sqrt{\alpha}) & \cosh (2 \kappa d \sqrt{\alpha})
\end{array}\right)\left(\begin{array}{c}
\psi \\
\left(\frac{1}{\kappa} \frac{d}{d x}-i A\right) \psi
\end{array}\right)\right|_{-d_{+}} .
\end{aligned}
$$

Finally, using the interface conditions (4.8), we find

$$
\begin{aligned}
& \left.\left(\begin{array}{c}
\psi \\
\left(\frac{1}{\kappa} \frac{d}{d x}-i A\right) \psi
\end{array}\right)\right|_{d_{+}} \\
& =\left.\left(\begin{array}{cc}
\cosh (2 \kappa d \sqrt{\alpha}) & \left(m_{n} / \sqrt{\alpha}\right) \sinh (2 \kappa d \sqrt{\alpha}) \\
\left(m_{s_{2}} \sqrt{\alpha} / m_{n}\right) \sinh (2 \kappa d \sqrt{\alpha}) & m_{s_{2}} \cosh (2 \kappa d \sqrt{\alpha})
\end{array}\right)\left(\begin{array}{c}
\psi \\
\left(\frac{1}{\kappa} \frac{d}{d x}-i A\right) \psi
\end{array}\right)\right|_{-d_{-}},
\end{aligned}
$$

which is the de Gennes condition (4.1) with

$$
\left(\begin{array}{ll}
M_{11} & M_{12} \\
M_{21} & M_{22}
\end{array}\right)=\left(\begin{array}{cc}
\cosh (2 \kappa d \sqrt{\alpha}) & \left(m_{n} / \sqrt{\alpha}\right) \sinh (2 \kappa d \sqrt{\alpha}) \\
\left(m_{s_{2}} \sqrt{\alpha} / m_{n}\right) \sinh (2 \kappa d \sqrt{\alpha}) & m_{s_{2}} \cosh (2 \kappa d \sqrt{\alpha})
\end{array}\right) .
$$

Note that the junction is symmetric if and only if $m_{s_{2}}=1$, i.e., whenever the two superconductors are the same. Also, note that in this case, (4.3) is satisfied. From (4.2), we then expect the supercurrent through the junction to be given by

$$
J=\frac{\sqrt{\alpha}}{m_{n} \sinh (2 \kappa d \sqrt{\alpha})}\left|\psi\left(d_{+}\right) \psi\left(-d_{-}\right)\right| \sin \left(\chi\left(d_{+}\right)-\chi\left(-d_{-}\right)\right) .
$$


Let us verify this directly. In the normal region, we have from (4.4) that $\mathbf{J}=(J, 0,0)^{T}$ is given by

$$
\begin{aligned}
J & =-\frac{1}{m_{n}}\left\{\frac{i}{2 d \kappa}\left(\psi^{*} \frac{d \psi}{d X}-\psi \frac{d \psi^{*}}{d X}\right)+|\psi|^{2} A\right\} \\
& =-\frac{i}{2 d \kappa m_{n}}\left(\phi^{*} \frac{d \phi}{d X}-\phi \frac{d \phi^{*}}{d X}\right) \\
& =-\frac{i \sqrt{\alpha}}{2 m_{n}}\left(C^{*} D-C D^{*}\right) .
\end{aligned}
$$

Writing $C$ and $D$ in terms of $\phi(-1)$ and $\phi(+1)$ gives

$$
C=\frac{\phi(1)+\phi(-1)}{2 \cosh (\kappa d \sqrt{\alpha})}
$$

and

$$
D=\frac{\phi(1)-\phi(-1)}{2 \sinh (\kappa d \sqrt{\alpha})}
$$

Hence, indeed, we have that

$$
\begin{aligned}
J & =-\frac{i \sqrt{\alpha}}{2 m_{n} \sinh (2 \kappa d \sqrt{\alpha})}\left(\phi(1) \phi(-1)^{*}-\phi(1)^{*} \phi(-1)\right) \\
& =\frac{\sqrt{\alpha}}{m_{n} \sinh (2 \kappa d \sqrt{\alpha})}\left|\psi\left(d_{+}\right) \psi\left(-d_{-}\right)\right| \sin \left(\chi\left(d_{+}\right)-\chi\left(-d_{-}\right)\right) .
\end{aligned}
$$

We note that as $d \rightarrow 0, J=O\left(d^{-1}\right)$, so that the approximation of $\mathbf{A}$ by a constant is valid. We also note that, as expected, $J$ decreases exponentially as $d$ increases and as $\alpha$ increases. Furthermore, $J$ also decreases as $m_{n}$ increases. Since large $m_{n}$ corresponds to a highly insulating material, this is in agreement with the experimental observation that junctions made from insulating materials need to be thinner than junctions made from metals, in order to obtain the same tunneling current.

\section{Computational results}

We now present the results of some computational experiments based on the model of $\S 3$. These results were obtained using a biquadratic finite element discretization of (3.1)-(3.4) applied to various normal-superconductor configurations. Although in each case we give results for only a single grid, grid refinement studies were carried out to insure that the results displayed are converged to graphical accuracy.

First, we consider a square of unit (dimesionless) size of homogeneous superconducting material; thus, in this example there is no normal region. We subdivide the unit square into a $12 \times 12$ uniform grid. We set $\kappa=3$ and obtain Figure 5.1 for the level curves of the magnitude of the order parameter. Note the symmetric, diamond-shaped arrangement of the four vortices. 
FIG. 5.1. Level curves of the magnitude of the order parameter for a unit square sample of a homogeneous superconductor having $\kappa=3$.

Next, we consider the arrangement sketched in the left-hand picture in Figure 5.2. We have a square of unit size with the shaded square of side 0.3 placed so that its lower left-hand corner is located at the point $(0.6,0.6)$ within the larger square. (The origin is located at the lower-left hand corner of the larger square.) The unshaded region is superconducting, while the shaded region is normal with $\alpha=1$. Again, we choose a $12 \times 12$ grid and set $\kappa=3$ and obtain Figure 5.3 for the level curves of the magnitude of the order parameter. Note that one of the vortices is attracted, i.e., pinned, by the normal region; the positions of the other three vortices also change so that the configuration has minimum energy. Instead of the diamond-shaped pattern of Figure 5.1, we roughly have, in Figure 5.3, a square-shaped pattern that is symmetric about the line $x=y$.

Fig. 5.2. Normal (shaded) and superconducting (unshaded) regions for the computational examples. 
FIG. 5.3. Level curves of the magnitude of the order parameter for a unit square sample of a homogeneous superconductor having $\kappa=3$ and having an embedded square normal region as sketched in the left-hand picture of Figure 5.2.

Our next example is for a homogeneous superconducting rectangle of width 0.7 and unit height. There is no normal region. We choose a $10 \times 16$ grid and set $\kappa=6$ and obtain Figure 5.4 for the level curves of the magnitude of the order parameter. Note that there are six vortices arranged symmetrically about the center of the rectangle.

Next, consider the superconducting-normal-superconducting junction sketched in the right-hand picture of Figure 5.2. Here we have a layer of normal material of thickness 0.02 sandwiched between two superconducting regions. (The unshaded region is superconducting, while the shaded region is normal with $\alpha=1$.) The overall S-N-S configuration has width 0.7 and unit height. The left edge of the normal layer is located at a distance equal to 0.3 from the left edge of the overall sample. Again, we choose a $10 \times 16$ grid and set $\kappa=6$ and obtain Figure 5.5 for the level curves of the magnitude of the order parameter. Note that the vortices are all attracted, i.e., pinned, by the normal region. One should compare the arrangement of vortices in Figure 5.4 for which there is no normal region, with that of Figure 5.5 for which a thin vertical normal region is present.

These examples illustrate that our model is potentially very useful in computational studies involving samples containing both superconducting and normal materials. They also serve to illustrate the fact that the model can, by itself, account for the various types of interactions involving normal/superconducting materials. In a future paper we intend to give much more comprehensive computational results using our model, including examples of transient flux-pinning phenomena and more detailed studies of S-N-S junctions. 
FIG. 5.4. Level curves of the magnitude of the order parameter for a $0.7 \times 1.0$ rectangular sample of a homogeneous superconductor having $\kappa=6$.

FIG. 5.5. Level curves of the magnitude of the order parameter for a superconducting/normal/superconduting junction of the type sketched in the right-hand picture of Figure 5.2. The superconductor has $\kappa=6$, the overall sample size is $0.7 \times 1.0$, and the normal region has thickness equal to 0.02 and is located to the left of the vertical centerline of the sample. 


\section{REFERENCES}

[1] P. DeGennes, Superconductivity in Metals and Alloys, Benjamin, New York, 1966.

[2] V. Ginzburg and L. Landau, On the theory of superconductivity, Zh. Eksperim. i Teor. Fiz. 20 1950, 1064-1082. [English translation: Men of Physics: L. D. Landau, I, Ed. by D. ter Haar, Pergamon, Oxford, 1965, 138-167.]

[3] K. Likharev, Superconducting weak links Rev. Modern Phys. 51 1979, 101-159.

[4] M. Tinkham, Introduction to Superconductivity, McGraw-Hill, New York, 1975. 